\title{
$1 \quad$ Does no-till agriculture limit crop yields?
}

\section{Matteo Tanadini and Zia Mehrabi ${ }^{1}$}

3 No-till is an agricultural practice widely promoted by governments, development agencies, and

4 agricultural organisations worldwide. However, the costs and benefits to farmers adopting no-till

5 are hotly debated ${ }^{1-4}$. Using a meta-analysis of unprecedented study size, Pittelkow et al. ${ }^{5}$ reported

6 that adopting no-till results in average yield losses of $-5.7 \%$, but that these losses can be limited

7 with the added implementation of two additional conservation agriculture practices - crop rotation

8 and crop residue retention, and in dry environments. They claimed that, as a result, resource

9 limited smallholder farmers, that are unable to implement the whole suite of conservation

10 agriculture practices are likely to experience yield losses under no-till. In a re-evaluation of their

11 analysis, we found that they overly biased their results toward showing that no-till negatively

12 impacts yields, and overlooked the practical significance of their findings. Strikingly, we find that

13 all of the variables they used in their analysis (e.g. crop residue management, rotation, site aridity

14 and study duration) are not much better than random for explaining the effect of no-till on crop

15 yields. Our results suggest that their meta-analysis cannot be used as the basis for evidence-based

16 decision-making in the agricultural community.

17

18 There is never a perfect analysis, and every analysis involves a large number of decisions, some

19 of which are often arbitrary. However, our re-evaluation found four major issues with Pittelkow

20 et al.'s meta-analysis, which are fundamental, based on standard statistical theory and best

21 practice. We show how methodologically accounting for these issues produces results that

22 strongly challenge the claims and conclusions of Pittelkow et al.

${ }^{1}$ Liu Institute For Global Issues, Institute for Resources, Environment and Sustainability, \& Centre for Sustainable Food Systems, University of British Columbia, Vancouver, British Colombia, Canada, V6T $1 \mathrm{Z2}$ 
First, we found that Pittelkow et al.'s estimates were biased to showing no-till leads to a yield loss. To obtain their $-5.7 \%$ yield loss figure, they log transformed the ratio of crop yield under no till and conventional till for each experiment, calculated a weighted mean effect of no-till for all these experiments and then backtransformed the result using the exponential function. However, due to Jensen's Inequality, using the exponential function to backtransform a log-ratio is both biased and inconsistent. In practical terms, this means that the exponentially back-transformed estimates are underestimated (i.e. too small), even when the sample size is very large. The magnitude of the bias depends on the variance of the random variable itself, and in many cases is likely to be negligible. However, for the case of Pittelkow et al., there was massive variation in the ratio of yields under no-till and till, which forced large bias in their results towards showing a negative effect of no-till on crop yields. Simply calculating the weighted mean yield ratio in the untransformed scale produces a value of $-2.4 \%$, which is around half of the crop yield loss reported by Pittelkow et al. $(-5.7 \%)$.

Second, Pittelkow et al. arbitrarily chose to discard observations from their data. They removed all data points further away than 5 standard deviations from the weighted mean, representing about $0.5 \%$ of the observations, from their analysis. They did not state any reason for how the threshold of 5 standard deviations was chosen (we are also not aware of any such rule). Because of the long-tailedness of the data, the removal of these observations had an important effect on the results. Including the observations that had been arbitrarily removed from the data leads to a yield ratio of only $-1.2 \%, \sim 80 \%$ less severe than the figure originally reported. Moreover, removing these observations from the data also did not solve the problem of outliers - it simply created more of them (Supplementary Information A).

Third, we found that the statistical significance testing reported by Pittelkow et al. was invalid. To calculate the confidence intervals around, and statistical significance of the effect of no-till on 
crop yields Pittelkow et al. used randomization and bootstrapping techniques. A central assumption of the methods they used is the independence of observations ${ }^{6}$. However, the 5493 experiments in their dataset arose from 609 different studies and so are unlikely to be independent. Our analysis clearly shows that observations gathered in the same study are more similar than observations between studies (Supplementary Information A), which in turn invalidates Pittelkow et al.'s statistical tests. In other words, the statistical significance testing reported in their article should be disregarded. There are established ways to account for this nonindependence (Supplementary Information A). However, due to the large sample size of this data set, there is also considerable power to detect statistical significant results, even if practical significance is lacking.

Finally, we found that Pittelkow et al. overlooked the practical significance of their findings. In their paper, Pittelkow et al. acknowledged that there was variability in the effect of no-till on yields, and in turn acknowledged that there were statistical differences in the effects of no-till between combinations (or groups) of agricultural practices. However, they did not mention, or visualise, the huge variability in the effects of no-till within each of these groups, nor determine how well their analysis was able to explain this variation. This is problematic because determination of the variation explained by moderators in meta-analysis is a critical component of interpreting the size and statistical significance of their effects ${ }^{7}$. Whilst ignored by Pittelkow et $a l$. , an indication of the practical significance of moderators used in a meta-analysis can be assessed graphically, or by using a simple meta-analytic equivalent of the $R^{2}$ statistic $^{7-8}$.

In all, forty-three per cent of experiments in Pittelkow et al.'s data show the same or higher yields under no-till. Moreover, the majority of yield ratios (e.g. 95\% quantiles), lay between a halving in 
73 yields $(-51 \%)$, to a three quarter increase in yields $(+74 \%)$ under no-till. As shown graphically in

74 Figure 1a, this variation makes it very difficult to make any general claims about negative effects

75 of no-till on crop yield. When we assessed how well the moderators used by Pittelkow et al. (i.e.

76 crop residue management, rotation, site aridity and study duration) helped to explain this large

77 degree of variation, we found that they all performed extremely poorly. As clearly shown in

78 Figure 1b, the cumulative explanatory power of all of the moderators Pittlekow et al. used in their

79 analysis (that is, the combined knowledge crop rotation, residue management, site aridity, and

80 study duration for explaining the effect of no-till on yield) is close to zero, with an $R^{2}$ of 0.03 .

81 Thus, whilst Pittelkow et al. stated that crop rotation, residue management and aridity were

82 generally important factors moderating yields under no-till, we found no support for these

83 conclusions.

The poor association between the moderators used by Pittelkow et al. and the effect of no-till on crop yield, suggests it makes little sense to make the general claims that rotation, residue management or site aridity will help increase yields under no-till. It also makes little sense to base further claims on this finding: such that we might expect yield losses to smallholder farmers, such as those in sub-Saharan Africa, due to their inability to implement additional conservation agricultural management practices. However, the applicability of Pittelkow et al.'s original analyses to smallholders in sub-Saharan Africa should be questioned anyway, as observations

92 from this region only make up $\sim 6 \%$ of their data set, with the majority of observations (69\%)

93 coming from N. America, Europe \& New Zealand. 

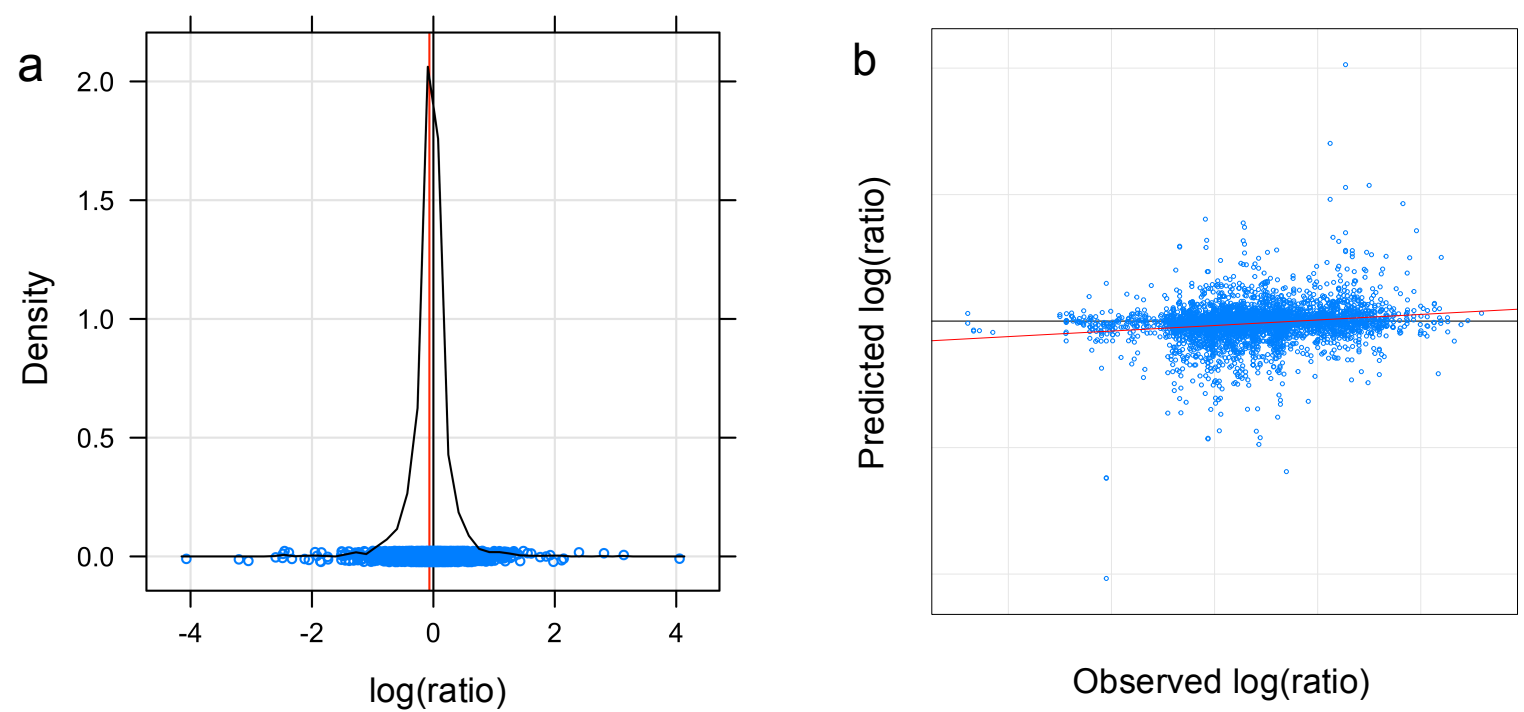

99 Figure 1 (a) This smoothed histogram (also called a density plot) shows the distribution of the log(ratio)

100 (i.e. the logarithm of the ratio between no-till and till yields). The weighted mean of the data (red line) can

101 hardly be distinguished from the null hypothesis of no difference between yields under no-till and till (black

102 line on zero). ( $n$ observations $=5492)$. (b) This graphical $R^{2}$ scatterplot illustrates the relationship between

103 the predicted and observed values of a linear model including all of the moderators used in the analysis of

104 Pittelkow et al. It shows that the crop rotation, crop residue management, aridity and study duration

105 moderators, as used by Pittelkow et al. as the basis for their analysis, poorly explain differences in the

106 effect of no-till on yields, with a cumulative explanatory power close to zero ( $n$ observations $=3753$; with a

107 reduction in $n$ due to the loss of observations from studies which failed to report on moderators, and so

108 couldn't be used to assess the interactions between moderators that formed the basis of Pittelkow et al.'s

109 main claims in their paper). 
112 In summary, our analysis suggests that Pittelkow et al.'s claim that no-till on average reduces

113 crop yields by $5.7 \%$ is incorrect. We found that mean yields under no-till are actually up to $80 \%$

114 less negative than those they reported $(-1.2 \%)$. This, in addition to the large variation in

115 experimental outcomes, and the poor explanatory power of the variables used by Pittelkow et al.

116 in their analysis, strongly challenges the central claims of their paper. Our analysis does not

117 corroborate their claim that farmers will experience yield deficits under no-till, or that rotation

118 and residue management will increase yields under no-till. With the large number of possible

119 factors that could drive differences in yields under no-till, we suggest that much more careful

120 attention be put towards better variable coding, model building, and model interpretation in future

121 if general statements on the outcomes of different management practices under no-till are to be

122 made.

123 There are of course, other, important reasons why farmers may wish to employ crop residue

124 management and crop rotation alongside no-till in dry environments (e.g. to control pests, soil

125 erosion and soil moisture retention). Similarly, there are other, potential limiting factors to the

126 uptake of no-till agriculture by smallholders in some regions of the world (e.g. access to

127 herbicides, implements) $)^{3-4}$. However, we found no support for the claim that yield losses are to be

128 expected for many of the smallholders currently adopting of no-till practices, or that rotation,

129 residue management or dry environments generally increase yields under no till. A wealth of

130 literature has already been published to try and aid biologists in making meta-analyses more

131 methodologically robust ${ }^{9-11}$. In an effort to try and further help future researchers working with

132 large datasets and meta-analysis, we detail methods for dealing with the issue we have outlined

133 here, and many additional others, which did not fit in this response in the Supplementary

134 Information accompanying this article. 


\section{Methods}

138 All methods, advice for dealing with other aspects of reproducibility and open science practice 139 relating to the original Pittelkow et al. article, such as data set formatting, code presentation, and 140 transparency of methods; and other guidance on treatment of large datasets, non-normality, non141 independence, and practical vs. statistical significance, are described in detail in the

142 Supplementary Information. We note that newer publications ${ }^{12-13}$ following the original Pittelkow 143 et al. article do not overcome the problems we have raised here.

145 Author contributions

146 MT \& ZM designed the study. MT wrote the first draft of the analysis. ZM wrote the first draft of 147 the paper. Revisions were undertaken by both MT \& ZM.

149 Competing financial interests

150 The authors declare no competing financial interests.

151 Corresponding author

152 Correspondence to: Zia Mehrabi (zia.mehrabi@ubc.ca) and Matteo Tanadini 153 (matteo.tanadini@gmail.com). 
164

165

166

167

168

169

170

171

172

173

174

175

176

177

178

179

180

181

182

183

184

185

186

187

188

189

190

191

192

193

194

195

196

197

\section{References}

1. Derpsch, R. et al. Why do we need to standardize no-tillage research? Soil and Tillage Research 137, 16-22 (2014).

2. Powlson, D. S. et al. Limited potential of no-till agriculture for climate change mitigation. Nature Climate Change 4, 678-683 (2014).

3. Stevenson, J. R., Serraj, R. \& Cassman, K. G. Evaluating conservation agriculture for small-scale farmers in Sub-Saharan Africa and South Asia. Agriculture, Ecosystems and Environment 187, 1-10 (2014).

4. Giller, K. E. et al. Beyond conservation agriculture. Frontiers in Plant Science 6, 1-14 (2015).

5. Pittelkow, C. M. et al. Productivity limits and potentials of the principles of conservation agriculture. Nature 517, 365-367 (2015).

6. Efron, B. \& Tibshirani, R.J. An introduction to the bootstrap. CRC press, (1994).

7. Lopez-Lopez, J.A. et al. Estimation of the predictive power of a model in mixed-effects meta-regression: A simulation study British Journal of Mathematical and Statistical Psychology 67, 30-48 (2014).

8. Aloe, A.M. et al. An alternative to $R^{2}$ for assessing linear models of effect size. Research Synthesis Methods 1, 272-283. (2010).

9. Koricheva, J. \& Gurevitch, J. Uses and misuses of meta-analysis in plant ecology. Journal of Ecology 102, 828-844 (2014).

10. Nakagawa, S. \& Santos, E.S.A. Methodological issues and advances in biological metaanalysis. Evolutionary Ecology, 26, 1253-1274. (2012)

11. Philibert, A., Loyce, C. \& Makowski, D Assessment of the quality of meta-analysis in agronomy. Agriculture Ecosystems \& Environment, 148, 72-82. (2012)

12. Pittelkow, C. M. et al. When does no-till yield more? A global meta-analysis. Field Crops Research 183, 156-168 (2015).

13. Lundy, M. E. et al. Nitrogen fertilization reduces yield declines following no-till adoption. Field Crops Research 183, 204-210 (2015). 